\title{
Distinguishing blood and lymph vessel invasion in breast cancer: a prospective immunohistochemical study
}

\author{
GG Van den Eynden ${ }^{1,2}$, I Van der Auwera',2, SJ Van Laere ${ }^{1,2}$, CG Colpaert ${ }^{1,2}$, P van Dam ${ }^{1,2}$, LY Dirix ${ }^{1,2}$, \\ PB Vermeulen ${ }^{*, 1,2}$ and EA Van Marck ${ }^{1,2}$
}

'Translational Cancer Research Group, Lab Pathology University of Antwerp/University Hospital Antwerp, Antwerp, Belgium; ${ }^{2}$ Translational Cancer Research Group, Oncology Center, General Hospital St-Augustinus, Oosterveldlaan 24, B-26 10 Wilrijk, Belgium

Recently, peritumoural (lympho)vascular invasion, assessed on haematoxylin-eosin (HE)-stained slides, was added to the St Gallen criteria for adjuvant treatment of patients with operable breast cancer (BC). New lymphatic endothelium-specific markers, such as D2-40, make it possible to distinguish between blood (BVI) and lymph vessel invasion (LVI). The aim of this prospective study was to quantify and compare BVI and LVI in a consecutive series of patients with BC. Three consecutive sections of all formalin-fixed paraffinembedded tissue blocks of 95 BC resection specimens were (immuno)histochemically stained in a fixed order: HE, anti-CD34 (panendothelium) and anti-D2-40 (lymphatic endothelium) antibodies. All vessels with vascular invasion were marked and relocated on the corresponding slides. Vascular invasion was assigned LVI $(C D 34 \oplus$ or $\ominus / D 2-40 \oplus)$ or BVI $(C D 34 \oplus / D 2-40 \ominus)$ and intra(contact with tumour cells or desmoplastic stroma) or peritumoural. The number of vessels with LVI and BVI as well as the number of tumour cells per embolus were counted. Results were correlated with clinico-pathological variables. Sixty-six (69.5\%) and 36 (37.9\%) patients had, respectively, LVI and BVI. The presence of 'vascular' invasion was missed on HE in 20\% (peritumourally) and 65\% (intratumourally) of cases. Although LVI and BVI were associated intratumourally $(P=0.02)$, only peritumoural $L V I$, and not $B V I$, was associated with the presence of lymph node $(L N)$ metastases ( $\left.p_{\text {peri }}=0.002\right)$. In multivariate analysis, peritumoural $L V I$ was the only independent determinant of LN metastases. Furthermore, the number of vessels with LVI was larger than the number of vessels with $\mathrm{BVI}(P=0.00 \mathrm{I})$ and lymphatic emboli were larger than blood vessel emboli $(P=0.004)$. We demonstrate that it is possible to distinguish between $\mathrm{BVI}$ and $\mathrm{LVI}$ in BC specimens using specific lymphatic endothelium markers. This is important to study the contribution of both processes to BC metastasis. Furthermore, immunohistochemical detection of lymphovascular invasion might be of value in clinical practice.

British Journal of Cancer (2006) 94, 1643-1649. doi: I0.1038/sj.bjc.6603 I 52 www.bjcancer.com

Published online 2 May 2006

(c) 2006 Cancer Research UK

Keywords: blood vessel invasion; lymph vessel invasion; breast cancer; D2-40

Breast cancer (BC) is the most frequent cause of death in women between 35 and 55 years of age (Uzzan et al, 2004). Loco-regional spread and recurrence of the disease can be debilitating, but metastasis to distant organs is the leading cause of breast cancer (BC)-related death. One of the very early steps in the metastatic cascade is (lympho)vascular invasion, or the penetration of tumour cells into lymph and/or blood vessels in and around the primary tumour. Therefore, tumour cell emboli in lymph and blood vessels are considered to be the morphological correlates of BC metastasising to loco-regional lymph nodes (LNs) and distant haematogenous sites, respectively. The presence of lymphovascular invasion indeed has been correlated to the presence of LN metastases and to poor prognosis in patients with BC (Sampat

*Correspondence: Dr PB Vermeulen, Department of Pathology, Oncology Center, General Hospital St Augustinus, Oosterveldlaan 24, B-26I0 Wilrijk, Belgium; E-mail: peter.vermeulen@gvagroup.be, URL: www.tcrg.be

Received 6 January 2006; revised 4 April 2006; accepted 5 April 2006; published online 2 May 2006 et al, 1977; Nealon et al, 1979, 1981; Dawson et al, 1982, 1986; Weigand et al, 1982; Bettelheim et al, 1984; Berger et al, 1988; Rosen et al, 1989, 1991; Lee et al, 1990; Clayton, 1991; Clemente et al, 1992; Neville et al, 1992; Fisher et al, 1993a,b; Lauria et al, 1995). Recently, interest in (lympho)vascular invasion has been increased owing to addition of peritumoural (lympho)vascular invasion to the St Gallen criteria for selection of adjuvant systemic treatment in operable BC (Goldhirsch et al, 2005). In these criteria, lymphovascular invasion is detected on haematoxylin-eosin (HE) sections that do not allow distinction between blood vessel invasion (BVI) and lymph vessel invasion (LVI).

Historically, different methodological problems have hampered visualisation of LVI and BVI in resection specimens of patients with BC. One of the major challenges has been to distinguish intratumoural lymph and blood vessels on $\mathrm{HE}$ slides from retraction artefacts caused by tissue fixation and processing. Most authors only included vessels with a clear-cut endothelium (Sampat et al, 1977; Nealon et al, 1979, 1981; Dawson et al, 1982, 1986; Weigand et al, 1982; Bettelheim et al, 1984; Berger et al, 1988; Rosen et al, 1989, 1991; Orbo et al, 1990; Clayton, 1991; Clemente 
et al, 1992; Neville et al, 1992; Fisher et al, 1993a,b; Pinder et al, 1994; Lauria et al, 1995), missing small and collapsed intratumoural vessels or vessels completely filled with tumour cells. Therefore, some authors only studied peritumoural LVI and BVI (Clemente et al, 1992; Neville et al, 1992; Lauria et al, 1995) or used morphological and topographical criteria to identify LVI (Orbo et al, 1990).

Another methodological problem has been distinguishing between blood and lymph vessels. Until recently, the lack of lymph vessel-specific markers made it very difficult to specifically study BVI or LVI. Therefore, some authors investigated 'vascular invasion', including both LVI and BVI (Berger et al, 1988; Pinder et al, 1994). Others tried to distinguish between both using blood vessel-specific characteristics such as typical blood vessel morphology or the presence of red blood cells or fibrin cloths (Weigand et al, 1982; Lauria et al, 1995). Lee et al $(1986,1990)$ used morphologic and immunohistochemical criteria and more recently, blood vessels were identified based on FVIII-antigen immunohistochemical or van Gieson elastica stains (Kato et al, 2002, 2003).

During the last decade, several specific markers for lymphatic endothelium have been discovered, such as Prox-1, a transcription factor, Lyve-1, a hyaluronan receptor, podoplanin, a glomerular podocyte membrane protein and D2-40. Recently, it has been shown that the D2-40 antibody specifically recognises podoplanin (Schacht et al, 2005) and that D2-40 and podoplanin are the most sensitive and specific antibodies for the detection of lymphatic endothelium (Evangelou et $a l$, 2005). In BC, we previously demonstrated that D2-40 is the best marker for lymphatic endothelium (Van der Auwera et al, 2005). Used in combination with panendothelial markers such as CD34 or CD31, it is now possible to differentiate between BVI and LVI and to study the role of both processes in BC metastasis. Recent data (reviewed by Pantel et al) have supported BC metastasis models hypothesising that lymphatic and haematogenous dissemination in $\mathrm{BC}$ are two complementary and specific pathways (Pantel and Brakenhoff, 2004). Therefore, the aim of this study was to evaluate a technique using a combined immunohistochemical expression profile to differentiate on an individual vessel basis between LVI and BVI in a consecutive series of patients with BC. Furthermore, we obtained quantitative data on LVI and BVI in BC and correlated the presence of LVI and BVI to other clinico-pathological variables.

\section{MATERIALS AND METHODS}

\section{Patient selection}

From 18 March 2005 until 19 August 2005, all tumourectomy, quadrantectomy or (modified) mastectomy specimens containing invasive $\mathrm{BC}$ examined at the Department of Pathology, General Hospital AZ St-Augustinus, Wilrijk, Belgium, were prospectively included in this study after written informed consent. As the investigators had no access to clinico-pathological data, all 109 included resection specimens were processed and analysed as described below. When the anonymisation code was broken, one patient with documented distant metastases and five patients with a local recurrence were excluded. From eight patients with more than one resection specimen, only the first was taken into account. Therefore, 95 patients with operable breast carcinoma were included in this study. One patient refused to have an axillary lymphadenectomy, hence no information on LN status was available in this patient. Table 1 summarises the clinicopathological data. Age, tumoural $(\mathrm{T})$ and nodal $(\mathrm{N})$ status, histological type, tumour grade, oestrogen (ER) and progesterone receptor (PR) status, p53 and HER2/neu oncoprotein status were recorded by review of the pathology files. Tumours were histologically graded according to the Nottingham modification
Table I Clinico-pathological data of patients included

\begin{tabular}{|c|c|}
\hline$N=95$ & Clinico-pathological data \\
\hline Mean age (years) & $60.5(33.5-86.1)$ \\
\hline \multicolumn{2}{|l|}{$T$ status } \\
\hline TI & 63 \\
\hline $\mathrm{T} 2$ & 27 \\
\hline T3 & 3 \\
\hline $\mathrm{T} 4$ & 2 \\
\hline \multicolumn{2}{|l|}{$N$ status } \\
\hline NO & 52 \\
\hline $\mathrm{NI}$ & 27 \\
\hline N2 & 10 \\
\hline N3 & 5 \\
\hline \multicolumn{2}{|l|}{ Histological type } \\
\hline Ductal & 72 \\
\hline Lobular & 10 \\
\hline Special type & 13 \\
\hline \multicolumn{2}{|l|}{ Tumour grade } \\
\hline 1 & 31 \\
\hline$\|$ & 35 \\
\hline III & 29 \\
\hline \multicolumn{2}{|l|}{ ER status } \\
\hline Negative & 25 \\
\hline Positive & 70 \\
\hline \multicolumn{2}{|l|}{ PR status } \\
\hline Negative & 32 \\
\hline Positive & 63 \\
\hline \multicolumn{2}{|l|}{ p53 status } \\
\hline Negative & 73 \\
\hline Positive & 22 \\
\hline \multicolumn{2}{|l|}{ HER2/neu status } \\
\hline Negative & 91 \\
\hline Positive & 4 \\
\hline \multicolumn{2}{|l|}{ Fibrotic focus } \\
\hline No & 56 \\
\hline$<1 / 3 r d$ tumour diameter & 20 \\
\hline$>1 / 3 r d$ tumour diameter & 19 \\
\hline \multicolumn{2}{|l|}{ Growth pattern } \\
\hline Infiltrative & 15 \\
\hline Mixed & 68 \\
\hline Expansive & 12 \\
\hline
\end{tabular}

of the Bloom and Richardson histological grading scheme (Bloom and Richardson, 1957; Elston, 1987). Tumoural and N status were assigned according to the tumour node metastasis classification of the American Joint Committee on Cancer (Green et al, 2002). Lymph nodes were examined according to the standard pathology procedure in our institution: HE step sections every $200 \mu \mathrm{m}$ for sentinel LNs and one HE section every $3 \mathrm{~mm}$ for non-sentinel LNs. No immunohistochemical techniques were used for detection of tumour cells in LNs. Furthermore, the presence and size of a fibrotic focus and the growth pattern of the tumour were assessed on the HE slides, as defined previously (Hasebe et al, 1998; Colpaert et al, 2001, 2003b). A fibrotic focus is defined as a scarlike area, consisting of fibroblasts and collagen fibres, that occupies various percentages of the centre of an invasive ductal carcinoma of the breast. In the infiltrative growth pattern, carcinoma cells infiltrate between pre-existing breast parenchymal structures, without significant disturbance of the breast architecture. In the expansive growth pattern, the tumour forms a well- 
circumscribed nodule consisting of carcinoma cells and desmoplastic connective tissue. Pre-existing breast parenchymal structures are not present inside the tumour but are pushed aside by the expansively growing nodule. The growth pattern is mixed infiltrative-expansive, when the tumour consists of a central expansive nodule surrounded by carcinoma cells showing an infiltrative growth pattern.

\section{(Immuno)histochemistry}

After routine histopathological examination, three consecutive $4 \mu \mathrm{m}$ sections of all formalin-fixed paraffin-embedded (FFPE) tissue blocks containing invasive carcinoma were cut and numbered. These slides were (immuno)histochemically stained in a fixed order: the first section of each block was stained with HE, the second with antibodies against CD34 (panendothelium marker) and the third with antibodies against D2-40 (lymphatic endothelium marker). The CD34 and D2-40 immunohistochemistry (IHC) were performed on the Dako Autostainer (Dako, Glostrüp, Denmark) using the Envision Dual Link + as the detection system (Dako). The CD34 (dilution 1/50, Clone Qbend10, Dako) and D2-40 (dilution 1/100, Dako) primary antibodies were both incubated for $30 \mathrm{~min}$.

\section{Assessment of blood and lymph vessel invasion}

First, all slides (HE, CD34 and D2-40) were screened for (lympho)vascular invasion using strict criteria (HE: tumour cells within an endothelium-lined vessel-like structure; CD34 or D2-40: tumour cells within an immunohistochemically positive vessel-like structure). Every slide was assessed by two investigators (GVdE and CC) without access to the data of other sections of the same resection specimen or other stainings of the same section. Every vessel with tumour cell invasion according to the criteria on one of the three consecutive sections was relocated on the other slides and assigned BVI or LVI based on the immunohistochemical staining profile (Figure 1). These blood and lymph vessels were also tracked on the corresponding HE stain and were scored as 'picked-up on HE' if the vascular invasion had also been marked on HE or 'missed on HE' if the vascular invasion had not been seen on HE. Tumour cell invasion in a vascular structure marked on the HE stain that was neither D2-40 nor CD34 positive was called 'overdiagnosis on HE'. Table 2 schematically shows the different possibilities. Furthermore, the foci of BVI and LVI were scored as intra- (in contact with tumour cells or desmoplastic stroma) or peritumoural and the size of every focus of BVI or LVI was assessed by counting the number of intravascular tumour cells.

\section{Statistical analysis}

Statistical analysis was performed with the SPSS 12.0 software package. A $P$-value $\leqslant 0.05$ was considered statistically significant, a $0.5<P$-value $\leqslant 0.1$ was considered a trend towards statistical significance. Normality was tested with a Kolmogorov-Smirnov test assuming normality of data if $P \geqslant 0.2$. As continuous data (e.g. number of vessels with LVI or BVI, size of lymphatic and blood vessel emboli) were not normally distributed, the median $(25-75$ th percentile) value is reported and compared between different groups with a Mann-Whitney $U$-test. For analysing associations between categorical variables (e.g. presence of LVI or BVI, presence of LN metastases, ER status and PR status), the $\chi^{2}$ test or - when the assumptions of the $\chi^{2}$ test were not met - the Fisher's exact test, was used. To build a multivariate model predicting axillary LN involvement, a logistic regression with backward procedure including intra- and peritumoural 'vascular' invasion, LVI and BVI was performed.
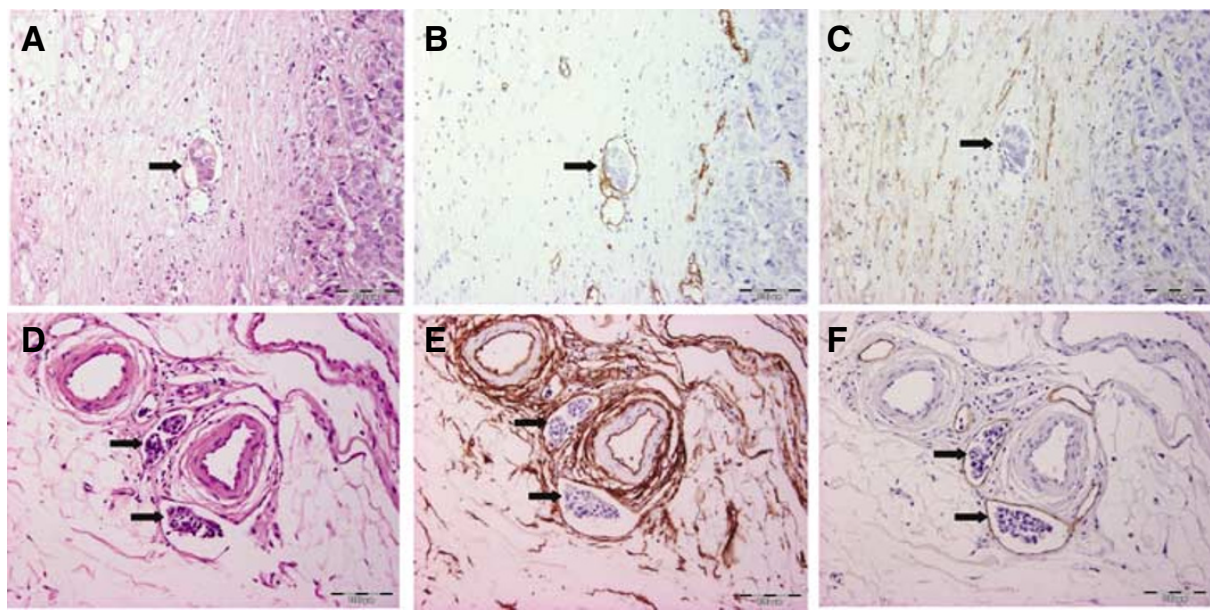

Figure I Overview of the histological and immunohistochemical stainings on consecutive slides, used to differentiate between BVI (upper row: $\mathbf{A}$, B and C) and LVI (lower row: D, E and F). Tumour cell emboli are indicated with black arrows. A and $\mathbf{D}$ : HE staining showing the presence of vascular invasion. B and E: On CD34 staining, both blood $(\mathbf{C})$ and lymph $(\mathbf{F})$ vessel endothelium stain positive. Furthermore, normal breast stromal cells are also CD34 positive (E). E and F: On D2-40 staining, the endothelium of vessels with BVI (B) and LVI (E) are respectively negative and positive. Desmoplastic stromal cells are also D2-40 positive $(\mathbf{C})$. (BVI= blood vessel invasion, $L V I=$ lymph vessel invasion).

Table 2 Decision table for the assessment of LVI or BVI based on the CD34/D2-40 staining profile of the vessel wal

\begin{tabular}{|c|c|c|}
\hline & D2-40- & D2-40+ \\
\hline $\begin{array}{l}\text { CD34- } \\
\text { CD34+ }\end{array}$ & $\begin{array}{l}\text { 'Overdiagnosis' on HE } \\
\text { Blood vessel 'Picked up' or 'missed' on HE }\end{array}$ & Lymph vessel 'Picked up' or 'missed' on HE \\
\hline
\end{tabular}

$\mathrm{BVI}=$ blood vessel invasion; $\mathrm{HE}=$ haematoxylin-eosin; $\mathrm{LVI}=$ lymph vessel invasion. 


\section{RESULTS}

\section{(Lympho)vascular invasion $v s$ number of blocks}

A total of 3297 vessels (661 intra and 2636 peritumoural) with LVI and 135 vessels with BVI (76 intra and 59 peritumoural) were demonstrated in consecutive sections of 446 FFPE tissue blocks. The median number of blocks per patient was four (range 1-20). The number of FFPE blocks investigated was significantly correlated with the size of the tumour $(\rho=0.40, P<0.001)$. Lymph vessel invasion $(P=0.01)$ and BVI $(P<0.001)$ were more frequently demonstrated in larger tumours and the number of vessels with LVI $(\rho=0.46, P<0.001)$ and BVI $(\rho=0.46, P<0.001)$ was correlated with the size of the tumour. The presence of LVI or BVI was not associated with the number of blocks investigated, but the number of vessels with LVI $(\rho=0.34, P=0.001)$, not BVI $(\rho=0.15, P=0.15)$, was correlated with the number of blocks.

\section{Blood vessel $v s$ lymph vessel invasion}

Lymph vessel invasion was more frequent than BVI. Sixty-six (69.5\%) patients had LVI (eight only intratumoural, 35 only peritumoural and 23 both intra- and peritumoural) and $36(37.9 \%)$ patients had BVI (12 only intratumoural, eight only peritumoural and 16 both intra- and peritumoural). In 28 (29.5\%) resection specimens, both LVI and BVI were found and in eight and 38 resection specimens, only BVI or LVI were found, respectively. The presence of LVI was associated with the presence of BVI intratumourally $(P=0.02)$, but not peritumourally $(P=0.11)$.

Furthermore, LVI was more extensive than BVI and lymphatic emboli were larger than blood vessel emboli. In seven cases, more than 100 vessels with LVI were found, whereas the maximum number of vessels with BVI was 16 . In cases with either LVI or BVI, the median numbers of lymph and blood vessels with vascular invasion were, respectively, six $(2-20.5)$ and three $(1-5.75)$
$(P=0.004)$ and the median sizes of the lymphatic and blood vessel emboli were $16(8-35)$ and eight $(5-11)$ intravascular tumour cells $(P=0.001)$. If both LVI and BVI were present, the median of the fraction of lymph vessels of the total number of vessels with vascular invasion was $80.0 \%$ (50.0-95.4\%). Figure 2 represents differences in extent and size between LVI and BVI. The median number of vessels with LVI in cases with both LVI and BVI was significantly higher than the median number of vessels with BVI in cases with both LVI and BVI $(P=0.003)$ or in cases with BVI only $(P=0.04)$. Furthermore, the median number of tumour cells/embolus was significantly higher in lymph vessel emboli than in blood vessel emboli, both in cases with LVI/BVI only $(P=0.03)$ or in cases with both LVI or BVI $(P=0.02)$.

\section{Immmunohistochemistry vs $\mathrm{HE}$}

On HE-stained sections it is impossible to differentiate between blood and lymph vessels. Therefore, the presence of 'vascular' invasion, including both BVI and LVI, was assessed. When only the results of this assessment were taken into account, $54(56.8 \%)$ patients had vascular invasion (five only intratumoural, 38 only peritumoural and 11 both intra- and peritumoural). Both intraand peritumourally, vascular invasion assessed on $\mathrm{HE}$ was associated with LVI $\left(p_{\text {intra }}=0.03, p_{\text {peri }}<0.001\right)$ and with BVI $\left(p_{\text {intra }}=0.01, p_{\text {peri }}=0.008\right)$. Nevertheless, the presence of LVI and BVI was missed intratumourally in 71.0 and $67.9 \%$ of immunohistochemically positive cases, respectively. Peritumourally, only 22.4 and $25 \%$ of cases with, respectively, LVI and BVI were missed on HE. Furthermore, not only the presence of LVI and BVI was underestimated on HE, but also the extent. On HE, vessels with BVI were more frequently missed than vessels with LVI: the median percentage of vessels with BVI and LVI seen on HE was, respectively, $0.0(0.0-31.3)$ and $26.8(0.0-54.0) \quad(P=0.009)$. Overdiagnosis of 'vascular' invasion on one or more HE-stained slides was seen in $23(24.2 \%)$ patients.

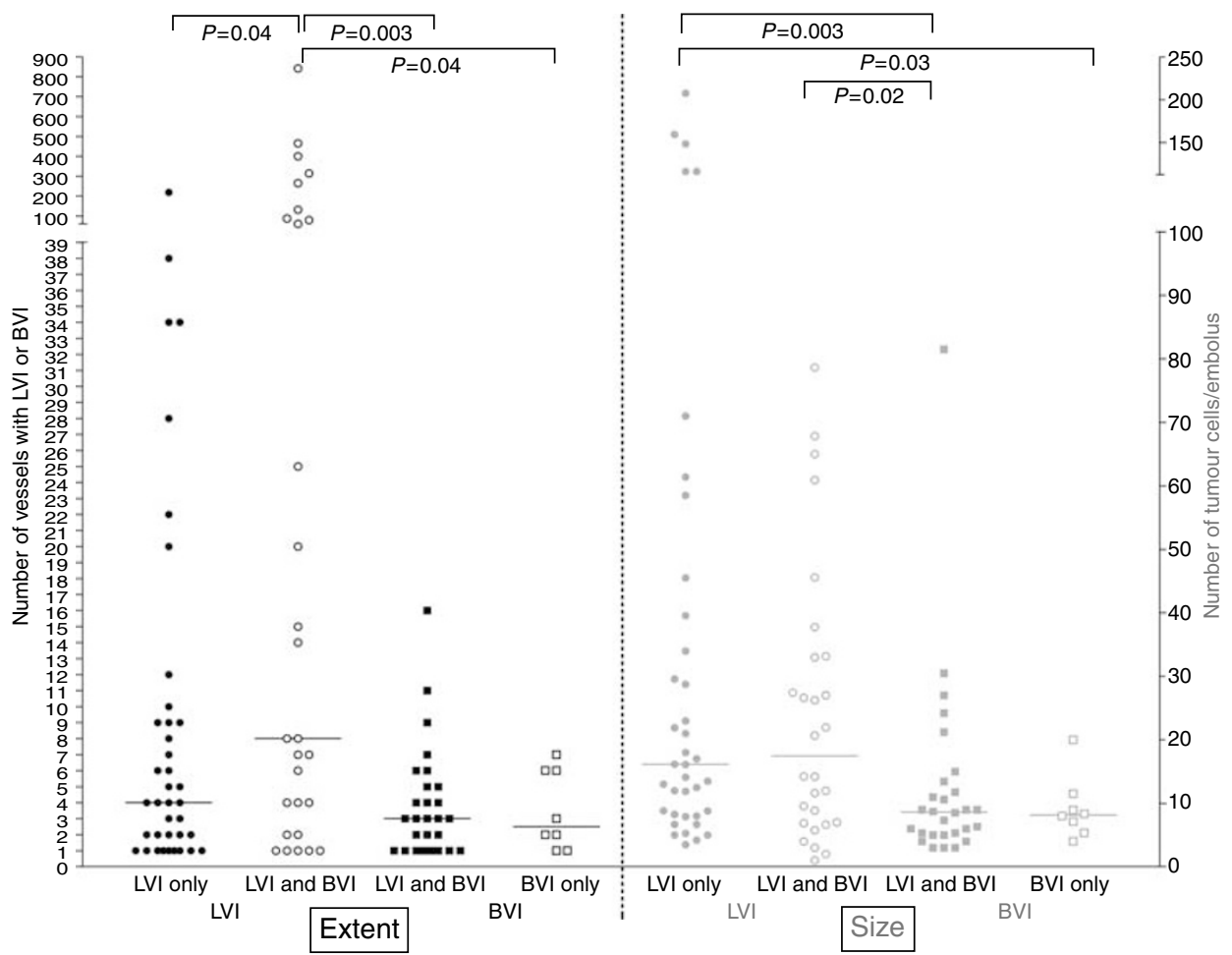

Figure 2 Differences in extent (left, black) and in size of tumour cell emboli (right, grey) between LVI (circles) and BVI (squares) in cases with LVI only (full circles), BVI only (open squares) or both LVI and BVI (open circles and full squares). The median value for each group is marked with a horizontal line and statistically significant differences are indicated on top ( $\mathrm{LVI}=$ lymph vessel invasion, $\mathrm{BVI}=$ blood vessel invasion). 
Correlation to LN metastases and other clinicopathological variables

The presence of peritumoural $(P=0.01)$ 'vascular' invasion on HE was associated with the presence of LN metastases. The presence of peritumoural LVI was strongly associated with the presence of LN metastases $(P=0.002)$. For intratumoural LVI, a trend to a positive association was found $(P=0.07)$. Neither the presence of intra-, nor the presence of peritumoural BVI was associated with LN involvement (Table 3). The negative predictive value for intratumoural 'vascular' invasion, peritumoural 'vascular'invasion, intratumoural LVI, peritumoural LVI, intratumoural BVI and peritumoural BVI were $60.3,68.0,61.9,75.0,57.6$ and $60 \%$, respectively. The positive predictive values were, respectively, 68.8, $57.1,58.1,56.5,58.1,50.0$ and $58.3 \%$. Multivariate logistic regression analysis revealed that peritumoural LVI $(\beta=1.38$, $P=0.003)$ was the most important determinant of axillary involvement. Table 4 represents the associations between the presence of LVI and BVI and other clinico-pathological variables.

\section{DISCUSSION}

We used the combination of the lymph endothelium-specific marker D2-40 and the panendothelial marker CD34 to detect and distinguish between LVI and BVI. LVI and BVI were found in 69.5 and $37.9 \%$ of patients, respectively. Other authors report LVI and especially BVI in BC to be less frequent: prevalences of LVI and BVI range from 8.8 (Nime et al, 1977) to $86 \%$ (Kahn and Marks, 2002) and from 4.2 (Lauria et al, 1995) to 33\% (Kato et al, 2002), respectively. The presence and extent of LVI and BVI correlated with the size of the tumour and only the extent of LVI correlated with the number of blocks investigated. Therefore, the high number of FFPE tissue blocks that was investigated per resection specimen cannot fully explain the increased frequency of LVI and BVI in this study. On the contrary, the use of IHC with endothelial markers to detect LVI and BVI accounts for their high prevalence.

Table 3 Cross-tabs showing the association between LVI (upper part) or BVI (lower part) and LN involvement

\begin{tabular}{|c|c|c|}
\hline & \multicolumn{2}{|c|}{ LN involvement } \\
\hline & No & Yes \\
\hline \multicolumn{3}{|c|}{ vascularinvasion (assessed on HE) } \\
\hline No & 47 & 31 \\
\hline Yes & 5 & 11 \\
\hline \multicolumn{3}{|c|}{ Peritumoural $(P=0.0 \mathrm{I})$} \\
\hline No & 31 & 14 \\
\hline Yes & 21 & 28 \\
\hline \multicolumn{3}{|c|}{ LVI (assessed on IHC) } \\
\hline \multicolumn{3}{|c|}{ Intratumoural $(P=0.07)$} \\
\hline No & 39 & 24 \\
\hline Yes & 13 & 18 \\
\hline \multicolumn{3}{|c|}{ Peritumoural $(P=0.002)$} \\
\hline No & 27 & 9 \\
\hline Yes & 25 & 33 \\
\hline \multicolumn{3}{|c|}{ BVI (assessed on $\mathbb{H C}$ ) } \\
\hline \multicolumn{3}{|c|}{ Intratumoural $(P=0.50)$} \\
\hline No & 38 & 28 \\
\hline Yes & 14 & 14 \\
\hline \multicolumn{3}{|c|}{ Peritumoural $(P=0.12)$} \\
\hline No & 42 & 28 \\
\hline Yes & 10 & 14 \\
\hline
\end{tabular}

$\mathrm{BVI}=$ blood vessel invasion; $\mathrm{HE}=$ haematoxylin-eosin; $\mathbb{I H C}=$ immunolistochemistry; $L N=$ lymph node; $L V I=$ lymph vessel invasion.
Immunohistochemistry is a very sensitive and specific technique. In our study, the presence of LVI or BVI was missed on HE in 71.0 and $67.9 \%$ of specimens intratumourally and in $20-25 \%$ of specimens peritumourally. Furthermore, also the extent of LVI and BVI was underestimated on HE. Differences in endothelial markers and in study populations further lead to differences in LVI and BVI prevalence. For LVI, the highest prevalences are found in the studies with D2-40 and podoplanin. Kahn et al demonstrated lymphatic invasion in $44 \%$ of LN negative and $86 \%$ of LN positive (overall 66\%) BC patients (Kahn and Marks, 2002). Recently, it has been shown that the D2-40 antibody specifically recognises podoplanin (Schacht et al, 2005) and that both antibodies against podoplanin and D2-40 can be regarded as reference standards for the identification of lymphatic vessels in most settings. Both

Table 4 Correlation between clinico-pathological variables and LVI and $\mathrm{BVI}$

\begin{tabular}{|c|c|c|c|c|c|c|}
\hline & \multicolumn{3}{|c|}{ LVI } & \multicolumn{3}{|c|}{ BVI } \\
\hline & Pos & Neg & & Pos & Neg & \\
\hline \multicolumn{7}{|l|}{ T status } \\
\hline TI & 39 & 24 & $p^{*}=0.03$ & 17 & 46 & $p *=0.00 \mathrm{I}$ \\
\hline $\mathrm{T} 2$ & 23 & 4 & & 17 & 10 & \\
\hline $\mathrm{T} 3$ & 2 & 1 & & I & 2 & \\
\hline $\mathrm{T} 4$ & 2 & 0 & & I & I & \\
\hline \multicolumn{7}{|l|}{$N$ status } \\
\hline No & 33 & 19 & $P=0.05$ & 16 & 36 & $P=0.14$ \\
\hline $\mathrm{NI}$ & 18 & 9 & & 11 & 16 & \\
\hline N2 & 10 & 0 & & 5 & 5 & \\
\hline N3 & 5 & 0 & & 4 & I & \\
\hline \multicolumn{7}{|l|}{ Histological type } \\
\hline IDA & 55 & 17 & $P=0.009$ & 32 & 40 & $P=0.07$ \\
\hline ILA & 3 & 7 & & 2 & 8 & \\
\hline Special Type & 8 & 5 & & 2 & 11 & \\
\hline \multicolumn{7}{|l|}{ Tumour grade } \\
\hline I & 15 & 16 & $P=0.005$ & 4 & 27 & $P<0.00$ \\
\hline 2 & 26 & 9 & & 12 & 23 & \\
\hline 3 & 25 & 4 & & 20 & 9 & \\
\hline \multicolumn{7}{|l|}{$E R$} \\
\hline Pos & 47 & 23 & $P=0.41$ & 25 & 45 & $P=0.46$ \\
\hline Neg & 19 & 6 & & 11 & 14 & \\
\hline \multicolumn{7}{|l|}{$P R$} \\
\hline Pos & 42 & 21 & $P=0.41$ & 21 & 42 & $P=0.20$ \\
\hline $\mathrm{Neg}$ & 24 & 8 & & 15 & 17 & \\
\hline \multicolumn{7}{|l|}{ p53 } \\
\hline Pos & 18 & 4 & $P=0.15$ & 10 & 12 & $P=0.40$ \\
\hline $\mathrm{Neg}$ & 48 & 25 & & 26 & 47 & \\
\hline \multicolumn{7}{|l|}{ HER2/neu } \\
\hline Pos & 3 & I & $P=1.00$ & 3 & I & $P=0.15$ \\
\hline $\mathrm{Neg}$ & 63 & 28 & & 33 & 58 & \\
\hline \multicolumn{7}{|l|}{ Fibrotic focus } \\
\hline Absent & 33 & 23 & $P=0.02$ & 15 & 41 & $P=0.005$ \\
\hline$<1 / 3$ th tumour diameter & 18 & 2 & & 8 & 12 & \\
\hline$>1 / 3$ th tumour diameter & 15 & 4 & & 13 & 6 & \\
\hline \multicolumn{7}{|l|}{ Growth pattern } \\
\hline Infiltrative & 9 & 6 & $P=0.66$ & 2 & 13 & $P=0.09$ \\
\hline Mixed & 48 & 20 & & 28 & 40 & \\
\hline Expansive & 9 & 3 & & 6 & 6 & \\
\hline
\end{tabular}

$\mathrm{BVI}=$ blood vessel invasion; $\mathrm{ER}=$ oestrogen receptor; $\mathrm{LVI}=$ lymph vessel invasion; $\mathrm{N}=$ nodal; $\quad \mathrm{Neg}=$ negative; $\quad \mathrm{Pos}=$ positive; $\quad \mathrm{PR}=$ progesterone receptor; $\mathrm{T}=$ tumoural. $P *$ : $P$-value of a $2 \times 2$ table only including $\mathrm{TI}$ and $\mathrm{T} 2$ tumours. 
anti-podoplanin and D2-40 antibodies have a sensitivity and specificity for lymphatic endothelium of over 95\% (Evangelou et al, 2005). We previously showed that in BC, D2-40 is the best marker for lymphatic endothelium (Van der Auwera et al, 2005). Most studies investigating BVI in BC used FVIII or a combination of FVIII and van Gieson elastica staining. Nevertheless, CD34 or CD31 is prefered to visualise blood vessels in solid tumours (Vermeulen et al, 1996, 2002), as FVIII expression is absent in some tumour capillaries that can lead to underestimation of the presence and extent of BVI (Vermeulen et al, 1995). As CD34 is not very specific for blood vessels, especially in tumour tissue, the combination with a specific lymphatic endothelium marker such as D2-40 is necessary to differentiate between LVI and BVI. In the present study, more than $50 \%$ of lymph vessels did show CD34 expression (data not shown). CD31 might be a more specific endothelial marker than CD34, nevertheless the latter was prefered in this study, as CD31 positivity of inflammatory cells might hamper interpretation.

Recently, peritumoural lymphovascular invasion has been included in the St Gallen guidelines for adjuvant therapy of operable breast carcinoma patients (Goldhirsch et al, 2005). In these criteria, peritumoural lymphovascular invasion is assessed on HE-stained sections during routine pathological examination, making it impossible to differentiate between LVI and BVI. In our data set, peritumoural 'vascular' invasion on HE and peritumoural LVI, not BVI were correlated with the presence of LN metastasis, still the only most important prognostic factor in BC. Multivariate analysis showed peritumoural LVI to be the most important determinant for the presence of LN metastases. Together with the increase in negative predictive value to $75 \%$ for peritumoural LVI, our results suggest that immunohistochemical detection of lymphovascular invasion and differentiation between LVI and BVI might be of value in clinical practice. Further studies are needed to address this issue.

If LVI and BVI are the morphological correlates of BC cells metastasising, respectively, via the lymphatic and the haematogenous route, our data support $\mathrm{BC}$ metastasis models stating that haematogenous and lymphatic metastasis are two complementary but specific metastasis pathways in BC. These newer models are in contrast with older models explaining BC metastasis as a stepwise cascade going from primary tumour via regional LNs to distant sites (Pantel and Brakenhoff, 2004). Only peritumoural LVI, and not BVI, was associated with the presence of LN metastases. Other authors found a correlation between BVI and LN metastases (Lauria et al, 1995; Kato et al, 2003). This might be due to the fact that the methods they used were too insensitive to discriminate between LVI and BVI, leading to misinterpretation of BVI as LVI. Another factor contributing to the correlation between BVI and LN status in some studies might be the association between LVI and
BVI. In our study, the presence of LVI was associated with the presence of BVI intratumourally, not peritumourally. As BVI is correlated with angiogenesis (Kato et al, 1999, 2001, 2003) and LVI is correlated with lymphangiogenesis (Schoppmann et al, 2004), the association between LVI and BVI might be due to the crossinteraction between lymphangiogenesis and hemangiogenesis. The hemangiogenic factors vascular endothelial growth factor (VEGF)A, basic fibroblast growth factor, angiopoietin-1 and -2 and platelet-derived growth factor have been shown to induce lymphangiogenesis and the lymphangiogenic factor VEGF-C can also induce hemangiogenesis (reviewed in Cao, 2005; Tammela et al, 2005). In a study of 29 invasive breast carcinomas, Choi et al (2005) reported a correlation between blood and lymph vessel microvessel density. The presence of a fibrotic focus is a surrogate marker for hypoxia-driven angiogenesis (Colpaert et al, 2003a) and for lymphangiogenesis in BC (Van der Auwera et al, 2005). In the present study, the presence of a fibrotic focus was indeed correlated with the presence of both LVI and BVI. The hypothesis that blood and lymph vessels are not just different routes that cancer cells can use to metastasise, but are characterised by a different biology is furthermore sustained by the fact that some patients exclusively show BVI or LVI and by the differences in size and number between LVI and BVI. In BVI, less vessels are involved and the size of the tumour emboli is smaller than in LVI. Very extensive vascular invasion is not found in BVI. To what extent these differences influence the metastatic capacity of both pathways remains to be elucidated.

In conclusion, we demonstrated that the described immunohistochemical technique made it possible to discriminate between BVI and LVI in BC and enabled a more sensitive detection of LVI and BVI and a better assessment of the extent of both than on conventional HE stains. Furthermore, our data demonstrate that most (lympho)vascular invasion in BC is LVI and that lymph vessel tumour emboli are larger than blood vessel tumour emboli. This suggests that LVI and BVI are not just different routes of BC metastasis, but that both pathways are characterised by a different biology.

\section{ACKNOWLEDGEMENTS}

G Van den Eynden is a research assistant of the Fund for Scientific Research Flanders. S Van Laere is a predoctoral assistant of the University of Antwerp. This work was supported by the Fund for Scientific Research Flanders Grant L.3.058.06N. We thank J Weyler for directing the statistical analysis and the technical staff of the Laboratory for Pathology of the GH St-Augustinus for expert technical assistance.

\section{REFERENCES}

Berger U, Bettelheim R, Mansi J, Easton D, Coombes R, Neville A (1988) The relationship between micrometastases in the bone marrow, histopathologic features of the primary tumor in breast cancer and prognosis. Am J Clin Pathol 90: 1

Bettelheim R, Penman H, Thornton-Jones H, Neville A (1984) Prognostic significance of peritumoral vascular invasion in breast cancer. $\mathrm{Br}$ Cancer 50: 771

Bloom H, Richardson W (1957) Histological grading and prognosis in breast cancer: a study of 1409 cases of which 359 have been followed for 15 years. Br J Cancer 11: 359

Cao Y (2005) Opinion: emerging mechanisms of tumour lymphangiogenesis and lymphatic metastasis. Nat Rev Cancer 5: 735

Choi W, Lewis M, Lawson D, Q Y-G, Birdsong G, Cotsonis G, Cohen C, Young A (2005) Angiogenic and lymphangiogenic microvessel density in breast carcinoma: correlation with clinicopathologic parameters and VEGF-family gene expression. Mod Pathol 18: 143
Clayton F (1991) Pathologic correlates of survival in 378 lymph nodenegative infiltrating ductal breast carcinomas. Mitotic count is the best single predictor. Cancer 68: 1309

Clemente C, Boracchi P, Andreola S, Del Vecchio M, Veronesi P, Rilke F (1992) Peritumoral lymphatic invasion in patients with node-negative mammary duct carcinoma. Cancer 69: 1396

Colpaert C, Vermeulen P, Van Beest P, Goovaerts G, Weyler J, Van Dam P, Dirix L, Van Marck E (2001) Intratumoral hypoxia resulting in the presence of a fibrotic focus is an independent predictor of early distant relapse in lymph node-negative breast cancer patients. Histopathology 39: 416

Colpaert C, Vermeulen P, Van Beest P, Soubry A, Goovaerts G, Dirix L, Harris A, Van Marck EA (2003b) Cutaneous breast cancer deposits show distinct growth patterns with different degrees of angiogenesis, hypoxia and fibrin deposition. Histopathology 42: 530 
Colpaert C, Vermeulen P, Fox S, Harris A, Dirix LY, Van Marck EA (2003a) The presence of a fibrotic focus in invasive breast carcinoma correlates with the expression of carbonic anhydrase IX and is a marker of hypoxia and poor prognosis. Breast Cancer Res Treat 81: 137

Dawson P, Ferguson D, Karrison T (1982) The pathological findings of breast cancer in patients surviving 25 years after radical mastectomy. Cancer 50: 2131

Dawson P, Karrison T, Ferguson D (1986) Histologic features associated with long-term survival in breast cancer. Hum Pathol 17: 1015

Elston CW (1987) Grading of invasive carcinoma of the breast. In Diagnostic Histopathology of the Breast, Page DL, Anderson TJ (eds), p 302. New York: Churchill Livingstone

Evangelou E, Kyzas PA, Trikalinos T (2005) Comparison of the diagnostic accuracy of lymphatic endothelium markers: Bayesian approach. Mod Pathol 18: 1490

Fisher E, Anderson S, Redmond C, Fisher B (1993a) Pathologic findings from the National Surgical Adjuvant Breast Project protocol B-06. 10year pathologic and clinical prognostic discriminants. Cancer 71: 2507

Fisher E, Costantino J, Fisher B, Redmond C (1993b) Pathologic findings from the National Surgical Adjuvant Breast Project (Protocol 4). Discriminants for 15-year survival. National Surgical Adjuvant Breast and Bowel Project Investigators. Cancer 71: 2141

Goldhirsch A, Glick J, Gelber R, Coates A, Thurlimann B, Senn H (2005) Meeting highlights: international expert consensus on the primary therapy of early breast cancer 2005. Ann Oncol 16: 1569

Green F, Balch C, Fleming I, Fritz A, Haller D, Morrow M, Page D (2002) AJCC Cancer Staging Handbook: 6/e TNM Classification of Malignant Tumors, p 140. New York: Springer-Verlag

Hasebe T, Tsuda H, Hirohashi S, Shimosato Y, Tsubono Y, Yamamoto H, Mukai K (1998) Fibrotic focus in infiltrating ductal carcinoma of the breast: a significant histopathological prognostic parameter for predicting the long-term survival of the patients. Breast Cancer Res Treat 49: 195

Kahn H, Marks A (2002) A new monoclonal antibody, D2-40, for detection of lymphatic invasion in primary tumors. Lab Invest 82: 1255

Kato T, Kameoka S, Kimura T, Nishikawa T, Kasajima T (2001) Angiogenesis and blood vessel invasion as prognostic indicators for node-negative breast cancer. Breast Cancer Res Treat 65: 203

Kato T, Kameoka S, Kimura T, Nishikawa T, Kobayashi M (2002) Blood vessel invasion as a predictor of long-term survival for Japanese patients with breast cancer. Breast Cancer Res Treat 73: 1

Kato T, Kameoka S, Kimura T, Nishikawa T, Kobayashi M (2003) The combination of angiogenesis and blood vessel invasion as a prognostic indicator in primary breast cancer. Br J Cancer 88: 1900

Kato T, Kimura T, Ishii N, Fujii A, Yamamoto K, Kameoka S, Nishikawa T, Kasajima $\mathrm{T}$ (1999) The methodology of quantitation of microvessel density and prognostic value of neovascularization associated with longterm survival in Japanese patients with breast cancer. Breast Cancer Res Treat 53: 19

Lauria R, Perrone F, Carlomagno C, De Laurentiis M, Morabito A, Gallo C, Varriale E, Pettinato G, Panico L, Petrella G, Et A (1995) The prognostic value of lymphatic and blood vessel invasion in operable breast cancer. Cancer 76: 1772

Lee A, Delellis R, Silverman M, Heatley GJ, Wolfe H (1990) Prognostic significance of peritumoral lymphatic and blood vessel invasion in nodenegative carcinoma of the breast. J Clin Oncol 8: 1457

Lee A, Delellis R, Silverman M, Wolfe H (1986) Lymphatic and blood vessel invasion in breast carcinoma: a useful prognostic indicator? Hum Pathol 17: 984

Nealon TJ, Nkongho A, Grossi C, Gillooley J (1979) Pathologic identification of poor prognosis stage I (T1N0M0) cancer of the breast. Ann Surg 190: 129
Nealon TJ, Nkongho A, Grossi C, Ward R, Nealon C, Gillooley J (1981) Treatment of early cancer of the breast (T1N0M0 and T2N0M0) on the basis of histologic characteristics. Surgery 89: 279

Neville A, Bettelheim R, Gelber RD, Save-Soderbergh J, Davis BW, Reed R, Torhorst J, Golouh R, Peterson H, Price K, Et A (1992) Factors predicting treatment responsiveness and prognosis in node-negative breast cancer. The International (Ludwig) Breast Cancer Study Group. J Clin Oncol 10: 696

Nime F, Rosen P, Thaler HT, Ashikari R, Urban J (1977) Prognostic significance of tumor emboli in intramammary lymphatics in patients with mammary carcinoma. Am J Surg Pathol 1: 25

Orbo A, Stalsberg H, Kunde D (1990) Topographic criteria in the diagnosis of tumor emboli in intramammary lymphatics. Cancer 66: 972

Pantel K, Brakenhoff R (2004) Dissecting the metastatic cascade. Nat Rev Cancer 4: 448

Pinder S, Ellis I, Galea M, O'rouke S, Blamey RW, Elston C (1994) Pathological prognostic factors in breast cancer. III. Vascular invasion: relationship with recurrence and survival in a large study with long-term follow-up. Histopathology 24: 41

Rosen P, Groshen S, Kinne DW (1991) Prognosis in T2N0M0 stage I breast carcinoma: a 20-year follow-up study. I Clin Oncol 9: 1650

Rosen PP, Groshen S, Saigo P, Kinne DW, Hellman S (1989) Pathological prognostic factors in stage I (T1N0M0) and stage II (T1N1M0) breast carcinoma: a study of 644 patients with median follow-up of 18 years. J Clin Oncol 7: 1239

Sampat M, Sirsat M, Gangadharan P (1977) Prognostic significance of blood vessel invasion in carcinoma of the breast in women. J Surg Oncol 9: 623

Schacht V, Dadras SS, Johnson LA, Jackson DG, Hong Y, Detmar M (2005) Up-regulation of the lymphatic marker podoplanin, a mucin-type transmembrane glycoprotein, in human squamous cell carcinomas and germ cell tumors. Am J Pathol 166: 913

Schoppmann S, Bayer G, Aumayr K, Taucher S, Geleff S, Rudas M, Kubista E, Hausmaninger H, Samonigg H, Gnant M, Jakesz R, Horvat R (2004) Prognostic value of lymphangiogenesis and lymphovascular invasion in invasive breast cancer. Ann Surg 240: 306

Tammela T, Petrova TV, Alitalo K (2005) Molecular lymphangiogenesis: new players. Trends Cell Biol 15: 434

Uzzan B, Nicolas P, Cucherat M, Perret GY (2004) Microvessel density as a prognostic factor in women with breast cancer: a systematic review of the literature and meta-analysis. Cancer Res 64: 2941

Van Der Auwera I, Van Den Eynden GG, Colpaert C, Van Laere SJ, Van Dam P, Van Marck EA, Dirix L, Vermeulen P (2005) Tumor lymphangiogenesis in inflammatory breast carcinoma: a histomorphometric study. Clin Cancer Res 11: 7637

Vermeulen P, Gasparini G, Fox S, Colpaert C, Marson L, Gion M, Belien J, De Waal RM, Van Marck E, Magnani E, Weidner N, Harris A, Dirix LY (2002) Second international consensus on the methodology and criteria of evaluation of angiogenesis quantification in solid human tumours. Eur I Cancer 38: 1564

Vermeulen P, Gasparini G, Fox S, Toi M, Martin L, Mcculloch P, Pezzella F, Viale G, Weidner N, Harris A, Dirix LY (1996) Quantification of angiogenesis in solid human tumours: an international consensus on the methodology and criteria of evaluation. Eur J Cancer 32A: 2474

Vermeulen P, Verhoeven D, Fierens H, Hubens G, Goovaerts G, Van Marck E, De Bruijn EA, Van Oosterom AT, Dirix LY (1995) Microvessel quantification in primary colorectal carcinoma: an immunohistochemical study. Br J Cancer 71: 340

Weigand R, Isenberg W, Russo J, Brennan MJ, Rich M (1982) Blood vessel invasion and axillary lymph node involvement as prognostic indicators for human breast cancer. Cancer 50: 962 